\title{
A closed biatrial procedure using bipolar radiofrequency ablation
}

Takeshi Miyairi, MD, ${ }^{a}$ Masayuki Nakao, MD, ${ }^{a}$ Ikutaro Kigawa, MD, ${ }^{a}$ Tadashi Kitamura, MD, ${ }^{a}$ Yujiro Miura, MD, ${ }^{a}$ Masaki Wakasugi, MD, ${ }^{\text {a }}$ Sachito Fukuda, MD, ${ }^{\text {a }}$ Dai Sonehara, MD, ${ }^{\mathrm{b}}$ and Hiroshi Nishimura, MD, ${ }^{\mathrm{c}}$ Tokyo, Japan

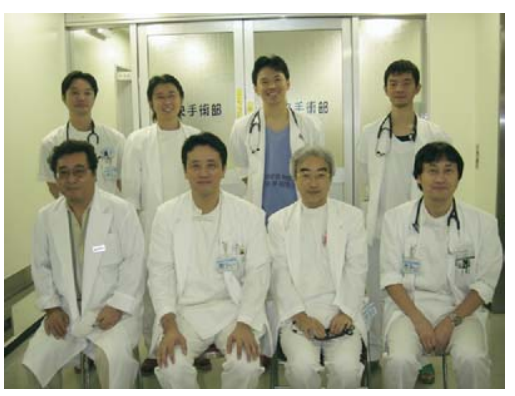

Drs Sonehara, Miyairi, Fukuda, Kigawa (front and Nakao (back row, left to right) radiofrequency ablation for atrial fibrillation without any incision being made on either atrium.

\section{Methods and Results}

Between December 2004 and April 2005, 4 patients with permanent atrial fibrillation undergoing heart surgery using cardiopulmonary bypass ( 2 aortic valve replacements, 1 coronary artery bypass grafting, 1 aortic valve replacement with coronary artery bypass grafting) underwent the biatrial procedure using bipolar radiofrequency $(\mathrm{RF})$ ablation (Atricure Inc, Cincinnati, Ohio) ${ }^{1}$ concomitantly. The average age of patients was $66 \pm 3$ years; all 4 patients were men. The mean and median durations of atrial fibrillation were $3.5 \pm 1$ years and 3 years, respectively. The mean diameter of the left atrium was $45 \pm 6 \mathrm{~mm}$.

The right atrial lesions were created and the right pulmonary veins (PVs) were isolated before establishment of cardiopulmonary bypass, whereas the left atrial lesions were created and the left PVs were isolated during cardiopulmonary bypass. To isolate the PVs, we clamped and ablated the rim of atrial tissue around the PVs as the device was passed around the PVs. To create the other lesions, we introduced the device through purse-string sutures in the atrial wall and manipulated it such that the interposing tissue between the inner and outer jaws was ablated. Each time the clamp was applied twice. Pacing to document transmural conduction block in the ablated lesions was not performed. The lesions were created in the following order (Figure 1). (1) Through a pursestring suture placed midway between the confluence of the superior and inferior venae cavae, lesions in the superior vena cava, the inferior vena cava, and the right atrial free wall were created. The right coronary artery was dissected and snared with a tape to avoid heat injury (Figure 2). (2) Through the right atrial appendage, the right atrial free wall was ablated both medially and

From the Departments of Cardiovascular Surgery ${ }^{\mathrm{a}}$ and Anesthesiology, Mitsui Memorial Hospital, and the Department of Cardiology, ${ }^{\mathrm{c}}$ Faculty of Medicine, University of Tokyo, Tokyo, Japan.

Received for publication Dec 8, 2005; revisions received Feb 15, 2006; accepted for publication March 6, 2006.

Address for reprints: Takeshi Miyairi, MD, Department of Cardiothoracic Surgery, Mitsui Memorial Hospital, Kandaizumicho 1, Chiyoda-ku, Tokyo 101-8643, Japan (E-mail: tmiyairi-tky@umin.ac.jp).

J Thorac Cardiovasc Surg 2006;132:168-9

$0022-5223 / \$ 32.00$

Copyright @ 2006 by The American Association for Thoracic Surgery doi:10.1016/j.jtcvs.2006.03.013 row, left to right); Wakasugi, Kitamura, Miura,

laterally. (3) The right and left PVs were isolated. (4) Through the left atrial appendage, the connecting lesion with the left PV isolation was created. The left atrial appendage was circumferentially oversewn. (5) Through the right upper PV, the roof of the left atrium below the superior vena cava and the ascending aorta was ablated. (6) Through the left upper PV, the roof of the left atrium was ablated, thus creating the connecting lesion between the right and left PV isolation line. (7) Finally, through a purse-string suture in the lower part of the left atrial wall, adjacent to the atrioventricular groove, the lines toward the right and left inferior PVs were ablated (Figure 3). The entire process using the bipolar RF device was completed in less than 30 minutes. The rest of the operative procedure was performed as indicated.

All patients survived the operation and were followed up in the outpatient clinic at a mean of $9 \pm 2$ months (6 months at the minimum). At the latest follow-up, all patients were receiving bepridil hydrochloride ( $200 \mathrm{mg} /$ day) given orally and were in normal sinus rhythm as demonstrated by electrocardiogram.

\section{Discussion}

Bipolar RF ablation was used to replace "cut and sew" lesions in the beating and then in the cardioplegically arrested heart. Except for several stabs to introduce the bipolar device, no incisions were made in either atrium.

With the right coronary artery snared and pulled, the bipolar RF device could safely be placed across the tricuspid valve. However, ablation to the mitral annulus could not be completed because RF

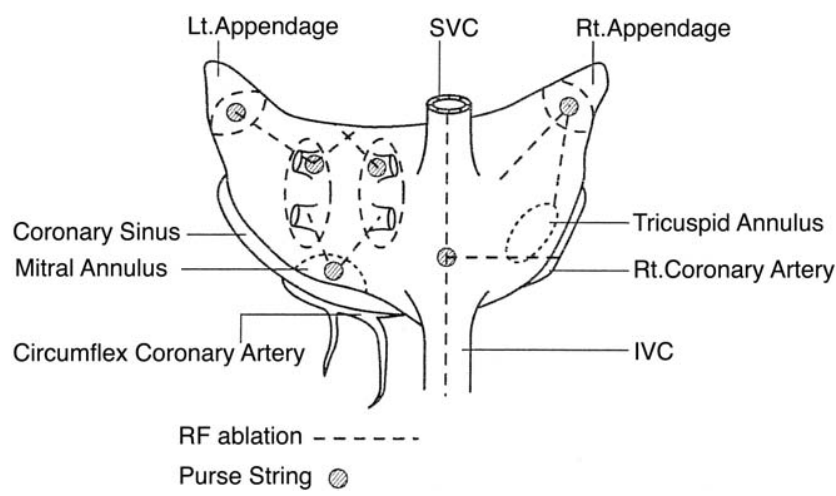

Figure 1. Diagram of all the ablations. SVC, Superior vena cava; $I V C$, inferior vena cava; $R F$, radiofrequency; $L t$, left; $R t$, right. 


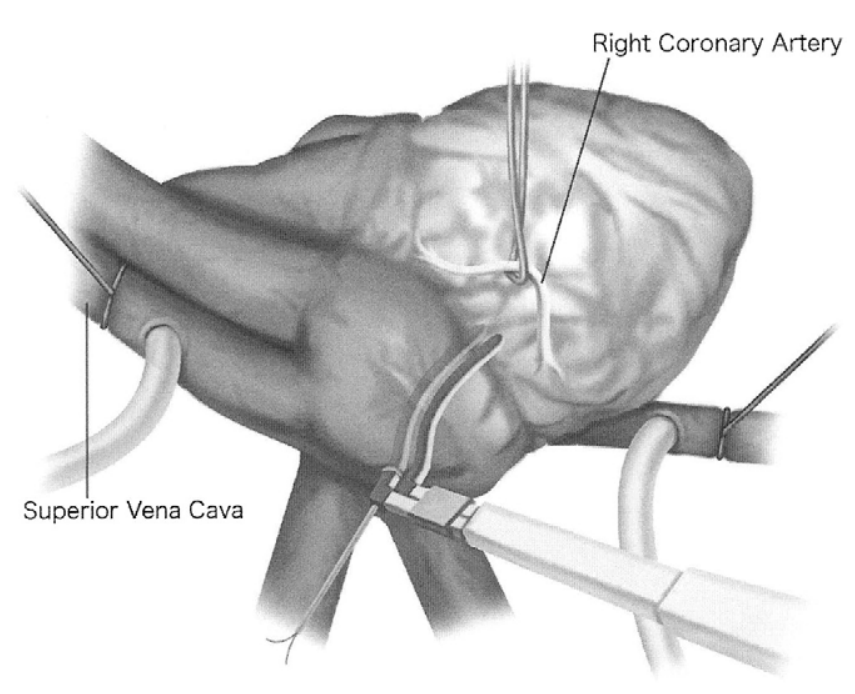

Figure 2. Right atrial free wall lesion crossing the atrioventricular groove.

energy might have injured the circumflex coronary artery. The optimal site for the device to cross the atrioventricular groove might be distal to the last circumflex marginal coronary artery. Although the septal lesion could not be ablated in this procedure, this lesion is believed to have only a small influence on the success rate of the procedure. ${ }^{2}$

The bipolar RF device appears to be safe and effective in creating transmural conduction block, replacing the standard surgical incisions. This device could be particularly beneficial for patients with atrial fibrillation who are undergoing a cardiac surgical procedure in which incision of the left atrium is not required, such as aortic valve replacement or coronary artery bypass grafting. However, this is a small series, with short follow-up, and all patients were still receiving antiarrhythmic drugs at latest followup. Thus, their drug-free success rate with this procedure was $0 \%$.

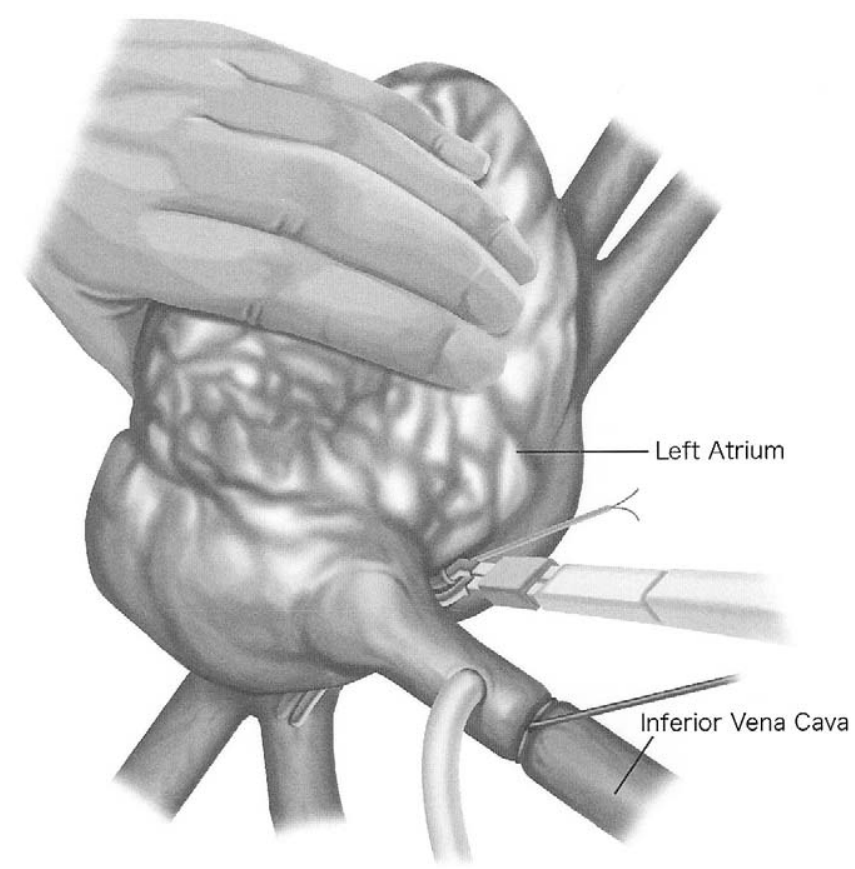

Figure 3. Right-sided lesion of the left atrium below the inferior vena cava.

Longer follow-up and more patients will be needed to assess the efficacy of this new approach.

\section{References}

1. Damiano, Jr RJ. Alternative energy sources for atrial ablation: judging the new technology. Ann Thorac Surg. 2003;75:329-30.

2. Gaynor SL, Ishii Y, Diodato MD, et al. Successful performance of Cox-Maze procedure on beating heart using bipolar radiofrequency ablation: a feasibility study in animals. Ann Thorac Surg. 2004;768: 1671-7. 